NASA Technical Memorandum 83710

\title{
Application of an Optimization Method to High Performance Propeller Designs
}

Li Ko Chang

Purdue University

West Lafayette, Indiana

and

George L. Stefko

Lewis Research Center

Cleveland, Ohio

Prepared for the

Twentieth Joint Propulsion Conference cosponsored by the AIAA, SAE, and ASME Cincinnati, Ohio June 11-13, 1984 



\title{
APPLICATION OF AN OPTIMIZATION METHOD TO HIGH PERFORMANCE PROPELLER DESIGNS
}

\author{
Li Ko Chang \\ Purdue University \\ West Lafayette, Indiana \\ and \\ George L. Stefko \\ National Aeronautics and Space Administration \\ Lewis Research Center \\ Cleveland, Ohio 44135
}

\section{Abstract}

The application of an optimization method to determine the propeller blade twist distribution which maximizes propeller efficiency is presented. The optimization employs a previously developed method which has been improved to include the effects of blade drag, camber and thickness. Before the optimization portion of the computer code is used, comparisons of calculated propeller efficiencies and power coefficients are made with experimental data for one NACA propeller at Mach numbers in the range of 0.24 to 0.50 and another NACA propeller at a Mach number of 0.71 to validate the propeller aerodynamic analysis portion of the computer code. Then comparisons of calculated propeller efficiencies for the optimized and the original propellers show the benefits of the optimization method in improving propeller performance. This method can be applied to the aerodynamic design of propellers having straight, swept, or nonplanar propeller blades.

\section{Introduction}

Optimization techniques are receiving increased attention as a method of solving real world problems and are being regarded as a unique tool for analysis, design, and selection processes. There are wide spread applications of optimization methods in science, engineering, and a variety of other fields. 1-5 In this paper, an optimization method is applied to maximize the propeller efficiency at a pre-specified design condition. Previous investigations for optimizing propeller twist used the method of calculus of variations 6,7 and a classical method. All of these approaches are formulated for straight propeller blades. The optimization method presented here in is an improved version of the method of Ref. 9, where it was demonstrated by analyzing propellers with tip devices that the method could be applied to complicated blade shapes. Features not available in the method of Ref. 9 but included in the optimization program used in this paper are the effects of blade drag, camber, and thickness. These additions improve the analys is and account for blade profile drag, thereby allowing the calculated efficiencies to be compared with experimental data.

The objective of this paper is to compare some calculated results of the propeller performance with the experimental data and then determine the maximum propeller efficiency by optimizing the blade twist distribution.
Description of the Propeller Analysis and the Optimization Method

The optimization computer code includes propeller performance analys is coupled with optimization procedures. In this paper, the computer code was applied to straight blade propellers with uniform inflow velocity field. However, the method can handle propellers having an arbitrary blade shape (swept or nonplanar propeller blades), any number of blades, and operating in a uniform or nonuniform flow field. The method currently works for a single rotating propeller but is being expanded to handle counter-rotating propellers. The performance analys is is based on the curved ifting 1 ine method. ${ }^{9}$ Summaries of the curved lifting line method and its use in the analysis of advanced propellers can be found in Refs. 10 to 12. Therefore, only a brief description of the curved lifting line method and the optimization procedures will be given herein. The propeller blade is replaced by a bound vortex and control points, which are located at the quarter chord point and three quarter chord point respectively. The propeller is modeled by blade elements, each of which has a blade angle, a chord length, a thickness ratio, and a design lift coefficient or camber. The varying vortex strength along the radius causes vorticity to be shed as helical vortex filaments. Influence coefficients are calculated, and the circulations are solved by satisfying the boundary condition at the control points. The lift coefficient of the blade at any radial location is then determined from the bound vortex strength at that radius. Drag is provided by correlated two dimensional airfoil data.

The optimization technique used was the 1970 version of SUMT (Sequential Unconstrained Minimization Technique) based on Fiafco and McCormick's penalty function method. 13,14 A variety of optimization methods are available, put the selection of the penalty function method 13,14
was due to its high reliability. The penalty function of Fiacco and McCormick can be stated as

$P(x, r)=f(x)+\frac{1}{r} \sum_{i=1}^{m} h_{j}^{2}(x)-r \sum_{j=1}^{k} \ln \left(g_{j}(x)\right)$,

where

$P(x, r) \quad$ penalty function

$x$ design vector (angle of twist of the blade elements)

$f(x)$ objective function (the negative value of the propeller efficiency) 
penalty parameter, a positive monotonjcally decreasing sequence, $r^{\ell}>r^{\ell+1}$ equality constraints

inequality constraints

total number of equality constraints

total number of inequality constraints

The problem is to minimize $P(x, r)$ by solving a sequence of unconstrained minimization problems. The search direction was calculated by the method of Davidon-Fletcher-Powe 11. 14-16 The opt imal step length is calculated by the golden section search 14 which locates the current minimum by regional eliminations. A complete description of the optimization method to obtain the best propeller blade twist is given in Ref. 9.

\section{Propeller Geometry}

Two NACA propellers which had experimental wind tunnel test data available were selected to compare the analytical and optimized results. The geometries of these two propellers are described below. The propeller blade geometries for the two propellers are shown in Fig. 1. Both of them were built with NACA 16 serjes airfoil sections. The lower speed propeller, 17 (Fig. 2) designated by NACA $10-(3)(08)-03$, is a two blade propeller. tested at speeds up to Mach number 0.64. Experimental results shown in Ref. 17 present the performance of this propeller in terms of apparent efficiency. However, because of the relatively low speed of the test data and the small size of the body aft the propeller, the difference between net and apparent efficiencies is sufficiently small to warrant the comparison to calculated net effi$c$ iency made here in.

The higher speed propeller, 18 (Fig. 3) designated by NACA $4-(3)(08)-045$, was tested for speeds up to Mach 0.73 . It is different from the NACA 10-(3) (08)-03 propeller mainly in the design lift coefficient and the chord length distribution.

For reference purposes, the NACA propeller designation numbering system (e.g., NACA $4-(3)(08)-045)$ is explained below. The number of the first group is the diameter in feet; the number of the second group (enclosed within the first set of parentheses) is the design lift coefficient (in tenths) of the blade section at the 0.7 -radius station; the number of the third group (enclosed within the second set of parentheses) is the thickness ratio of the blade section at the 0.7 -radius station; and the number of the fourth group is the blade solidity expressed as the ratio of the blade chord at the 0.7 -radius station to the circumference of the circle having a radius 0.7 of the propeller tip radius. The NACA 4-(3) (08)-045 propeller thus has a diameter of $4 \mathrm{ft}$ and the blade section at the 0.7 -radius station has a design lift coefficient of 0.3 , a thickness ratio of 0.08 , and a blade solidity of 0.045 .

\section{Aerodynamic Performance}

The computer code was first used, without the optimization procedure, to calculate the aerodynamic performance of the two propellers using the original twist distributions. The geometry of the lifting line used in the analysis was calculated using the geometry of the original design as shown in Fig. 2 for the lower speed propeller and Fig. 3 for the higher speed propeller.

For the lower speed propeller, the blade angle at 0.75 radius was specified in Ref. 17 to be $35^{\circ}$ for Mach numbers from 0.24 to 0.35 , and $45^{\circ}$ for Mach numbers from 0.35 to 0.50 . These Mach numbers correspond to advance ratio from 1.2 to 2.6 . The predicted results are compared with test data 17 in Figs. 4 and 5 . In Fig. 4 , the predicted power coefficient is compared with experimental data as a function of advance ratio. The comparison shows that both the shape and the level of the power coefficient are predicted very well. Differences are within one percent. Figure 5 presents the predicted and measured efficiencies. Again, the comparison shows that both the shape of the efficiency curve and the efficiency level agree well. The maximum difference is 1.5 percent.

The performance of the higher speed propeller was calculated at different values of advance ratio from 3.1 to 3.8 for a blade angle at 0.75 blade radius of $55^{\circ}$. The test Mach number for the higher speed propeller 18 was not corrected for tunnel wall interference. When this correction is made, the tunnel datum Mach number of 0.70 corresponds to a freestream Mach number of 0.71 . The analytical calculations were done at this freestream Mach number of 0.71 . In Fig. 6, the predicted power coefficient and the experimental data are compared. For lower values of advance ratio, the predictions agreed well with the data, but for advance ratios above 3.4 the predictions show a slight overestimate for power coefficient. A comparison of the predicted and experimental efficiency values for the higher speed propeller is shown in Fig. 7 . The predicted efficiency underestimates the data by about one percent for advance ratios below 3.5, and overpredicts the efficiency at advance ratios above 3.5 .

All these comparisons between the analytically predicted results and the test data demonstrate the capability of the code for performance analys is and validate its use for the optimization.

\section{Results of the 0ptimization}

Optimization performed on the lower speed and the higher speed propellers were based on a typical cruise condition for each propeller. For the lower speed propeller, the cruise condition was at a freestream Mach number of 0.44 , an advance ratio of 2.2 and a blade angle at the 0.75 radius of $45^{\circ}$. For the higher speed propeller, a typical cruise condition was at a freestream Mach number of 0.71 , an advance ratio of 3.3 and a blade angle at the 0.75 radius of $55^{\circ}$. For each propeller, the initial input of the blade twist for the optimization process was the twist of the original design. The equality constraint for the optimization process was the power coefficient at cruise.

In Fig. 8, the original and optirnized propeller blade twist distribution for the lower speed propeller is shown. The shape of the optimized twist and original design are almost the same.

Figure 9 shows the comparison of the propeller efficiencies that were calculated for the original and optimized lower speed propeller design. To show the magnitude of the drag loss, the ideal ef$f$ iciency was calculated by an option in the code 
and shown on this figure. The ideal efficiency accounts for the swirl loss, tip loss, and axial momentum loss but does not include the drag loss. The blade angle at the 0.75 radius was varied at a constant advance ratio so that the value of efficiency at different values of power coefficient could be obtained. As shown in Fig. 9, there is actually no improvement in efficiency by the optimization process. This lack of improvement is not surprising since there was almost no change in twist. It thus appears that the original propeller blade twist design was very good.

Figure 10 shows the twist distribution for the original design and the optimized blade of the higher speed propeller. The opt imized blade shows a different distribution than the original design, having higher relative blade angles very near the hub and in the outboard regions. Blade angles are smaller in the middle part of the optimized blade than in the original design. Thus the optimized propeller blade would have higher loading at the hub and over the outer 20 percent of the blade, but a lower loading across the mid-portion of the blade.

A comparison between the predicted efficiencies of the optimized and original blade designs is presented in Fig. 11. The comparison was made for different values of the power coefficients as Nas done in Fig. 9. The optimization process improved the higher speed propeller efficiency by about 1 percent at the cruise power coefficient of 0.18 . At lower power coefficients, improvements as large as 2 percent were predicted. Again, the ideal efficiency was added on the figure for a comparison of the blade drag. The larger drag losses on the higher speed propeller as compared with the lower speed propeller (Fig. 9) are due to the higher compressible drag caused by the much higher helical tip Mach numbers.

\section{Conclusions}

A method of maximizing propeller performance has been presented and demonstrated using the blade geometry of two NACA propellers. The method in$c$ ludes the effects of blade drag, camber, and thickness. Comparisons made in this paper show that the present method can be employed to perform propeller derodynamic design. Also, the opt imization method provides a tool for decreasing the time it takes to design a propeller. The paper presents the results of optimization of straight blade propellers but the method is equally capable of optimizing swept or other nonplanar propeller blades.

\section{References}

1. Vanderplaats, G. N. and Moses, F., "Structural Optimization by Methods of Feasible Directions," Computers and Structures, Vol. 3, JuTy 1973, pp. 739-755.

2. Hicks, R. and Henne, P. A., "Wing Design by Numerical Optimization," AIAA Paper 77-1247, Aug. 1977.
3. Ragsdell, K. M. and Phillips, D. T., "Optimal design of a Class of Welded Structures Using Geometric Programing," Journal of Engineering for Industry, VoI. 98, Aug. 1976, pp. 1021-1025.

4. Reinert, R. P., "Weight Optimization of Ultra Large Space Structures, "SAWE Paper 1301, May, 1979.

5. Vanderplaats, G. N., "A Robust Feasible Direction Algorithm for Design Synthes is, " Structures, Structural Dynamics and Materials Conference, Lake Tahoe, Nevada, May 2-4, 1983, pp. 392-399.

6. Lock, C. N. H., Pankhurst, R. C., and Fowler, R. G., "Determination of the Opt imum Twist of an Airscrew Blade by the Calculus of Variations," REM 2088, Jan. 1942.

7. Haines, A. B. and Diprose, K.V., "The Application of the Calculus of Variations to Propeller Design with Particular Reference to Spitfire VII with Merlin 61 Engine," ReM 2083, May 1943.

8. Moriya, T., "Formulae for Propeller Characteristics Calculation and a Method to Obtain the Best Pitch Distribution," Selected Scientific and Technical Papers, Moriya Memorial Committee, University of Tokyo, Aug. 1959, pp. 43-47.

9. Chang, L. K. and Sullivan, J. P. "Opt imization of Propeller Blade" Twist by An Analytical Method," AIAA Journal, Vol. 22, Feb. 1984, pp. 252-255.

10. Bober, L. J. and Mitchell, G. A., "Summary of Advanced Methods for Predicting High Speed Propel ler Performance," AIAA Paper 80-0225, Jan. 1980.

11. Stefko, G. L., Bober, L. J., and Neumann, H. E., "New Test Techniques and Analytical Procedures for Understanding the Behavior of Advanced Propellers," SAE Paper 830729, April 1983.

12. Bober, L. J. and Chang, L. K., "Factors Influencing the Predicted Performance of Advanced Propeller Designs," AIAA Paper 81-1564, July 1981 .

13. Fiacco, A. V. and McCormick, G. P., Nonlinear Programming: Sequential Unconstrained Minimization Techniques, Wiley, New York, 1968.

14. Gabriele, G. A. and Ragsdell, K. M., "OPTLIB: An Optimization Program Library," Purdue Research Foundation, June 1977.

15. Davidon, W. C., "Variable Metric Method for Minimization," Argonne National Lab Report, Argonne, IL, ANL-5990(Rev.), 1959.

16. Fletcher, R. and Powell, M.J.D. "A Rapid Convergent Descent Method for Minimization," Computer Journal, Vol. 6, July 1963, pp. 163-168.

17. Corson, B. W. Jr. and Maynard, J. D.," The Langley 2,000-Horsepower Propell ler Dynamometer and Tests at High Speed of An NACA 10-(3) (08) -03 Two-Blade Propeller, "NACA TN-2859, 1952.

18. Stack, J., Draley, E. C., Delano, J. 8., and Feldman, L., "Investigation of the NACA 4-(3) (08) -03 and NACA 4-(3)(08)-045 Two-Blade Propellers at Forward Mach Numbers to Determine the Effects of Compressibility and Solidity on Performance," NACA Report 999, 1944. 



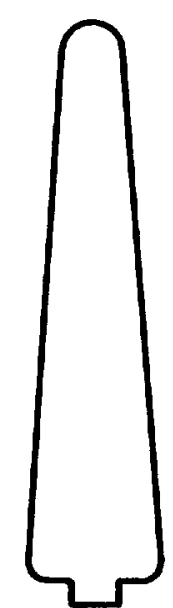

LOWER SPEED (LS) PROPELLER (NACA 10-(3) (08) -03)

(NACA TN 2859)

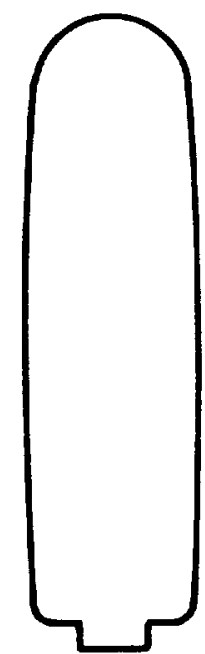

HIGHER SPEED (HS) PROPELLER

(NACA 4-(3) (08)-045)

(NACA REPORT 999)

Figure 1. - Propeller blade planforms used in the analysis.
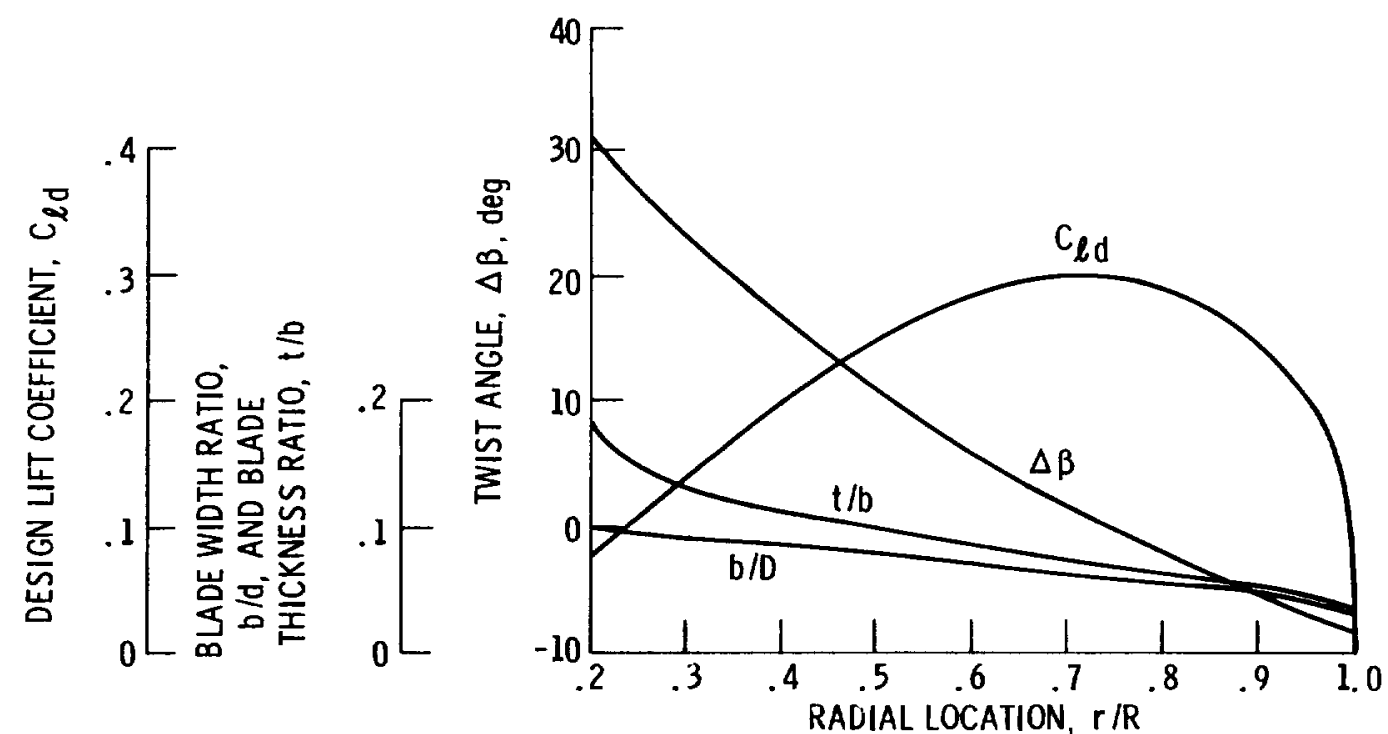

Figure 2. - Blade geometry for the lower speed (LS) propeller (NACA TN 2859). 

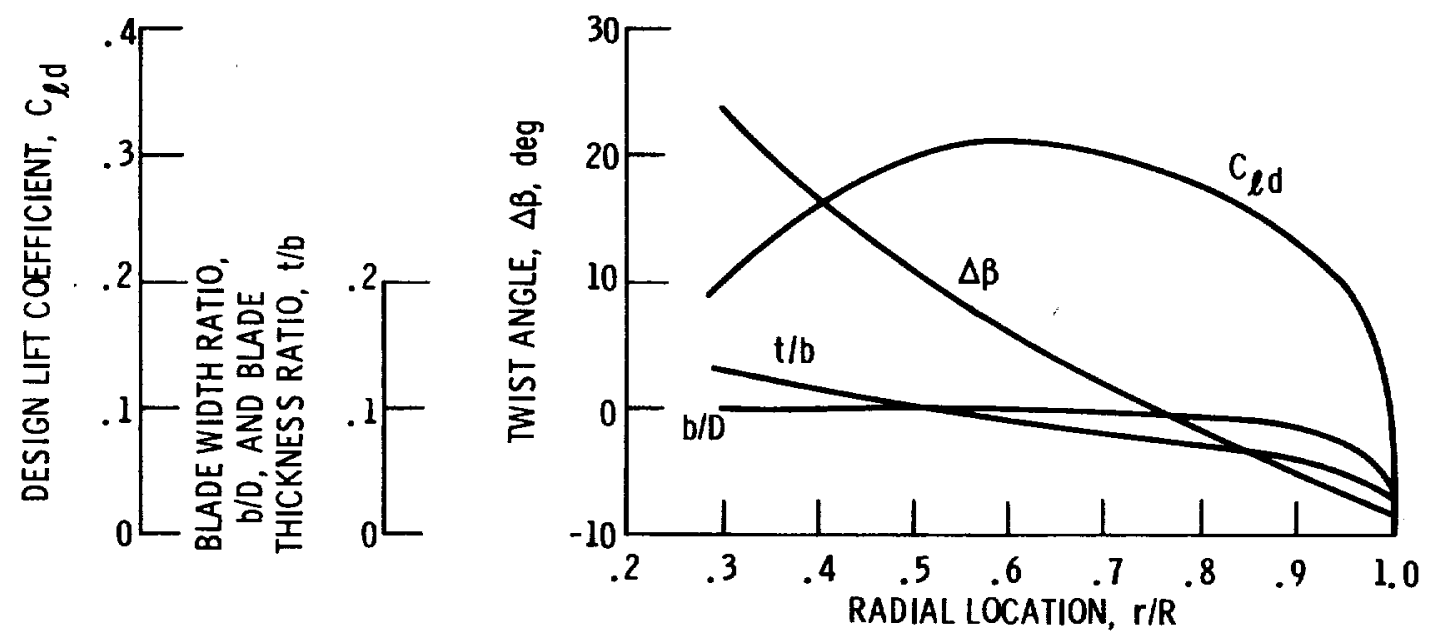

Figure 3. - Blade geometry for the higher speed (HS) propeller (NACA report 999).

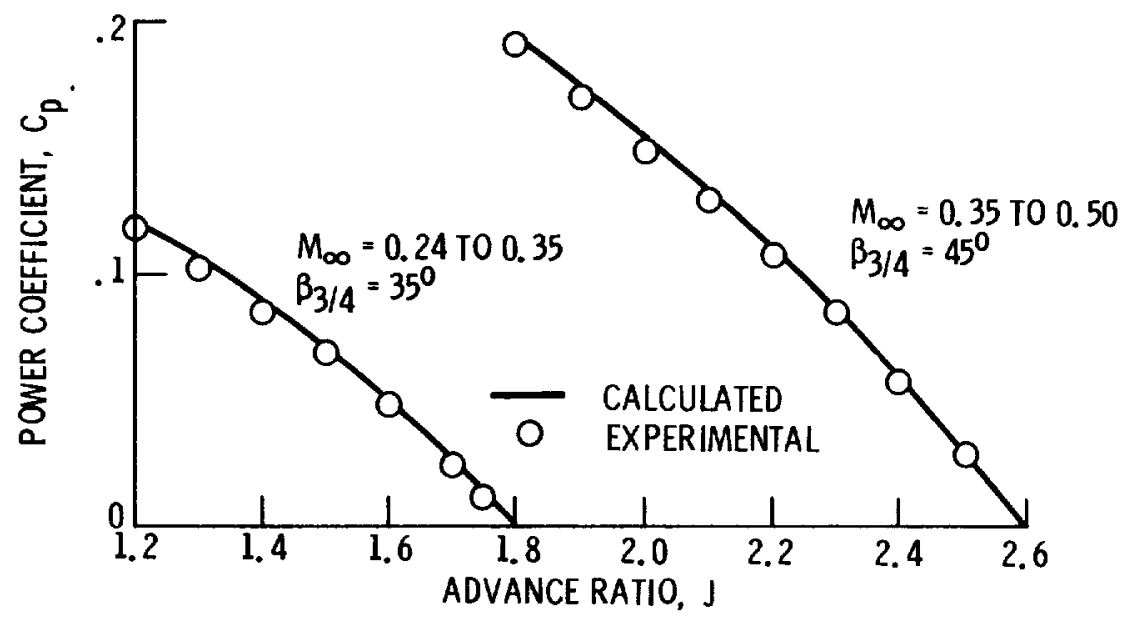

Figure 4. - Comparison of analytical and experimental power coefficients for the lower speed propeller. Two blade propeller; rotational speed, $1350 \mathrm{rpm}$. 


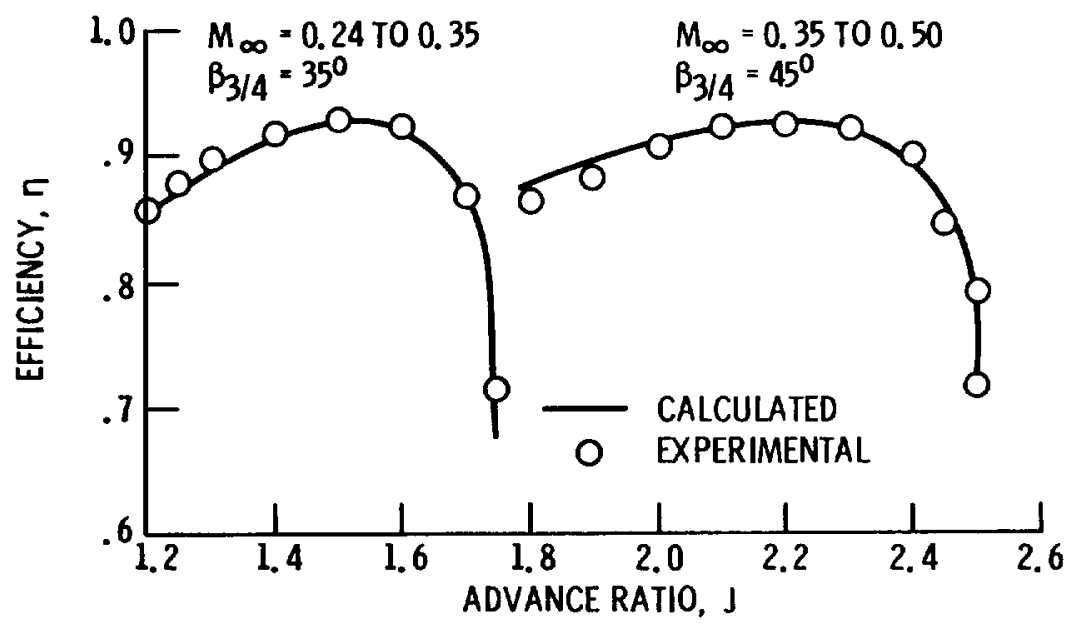

Figure 5. - Comparison of analytical and experimental apparent efficiencies for the lower speed propeller. Two blade propeller; rotational speed, $1350 \mathrm{rpm}$.

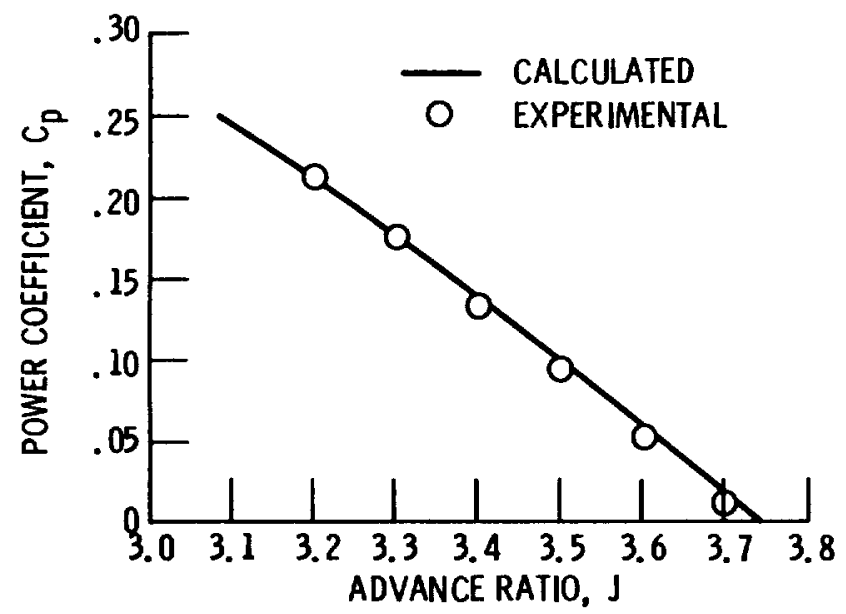

Figure 6. - Comparison of analytical and experimental power coefficients for the higher speed propeller. Freestream Mach number, 0.71; two blade propeller; blade angle at 0.75 radius, $55^{\circ}$. 


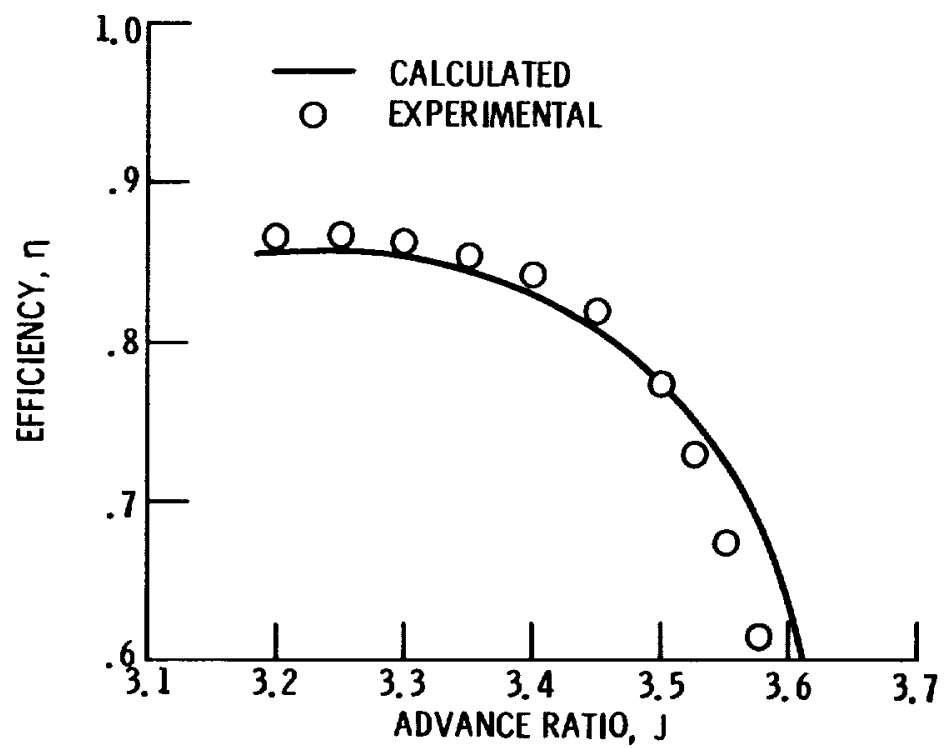

Figure 7. - Comparison of analytical and experimental net efficiencies for the higher speed propeller. Freestream Mach number, 0.71 ; two blade propeller: blade angle at 0.75 radius, $55^{\circ}$.

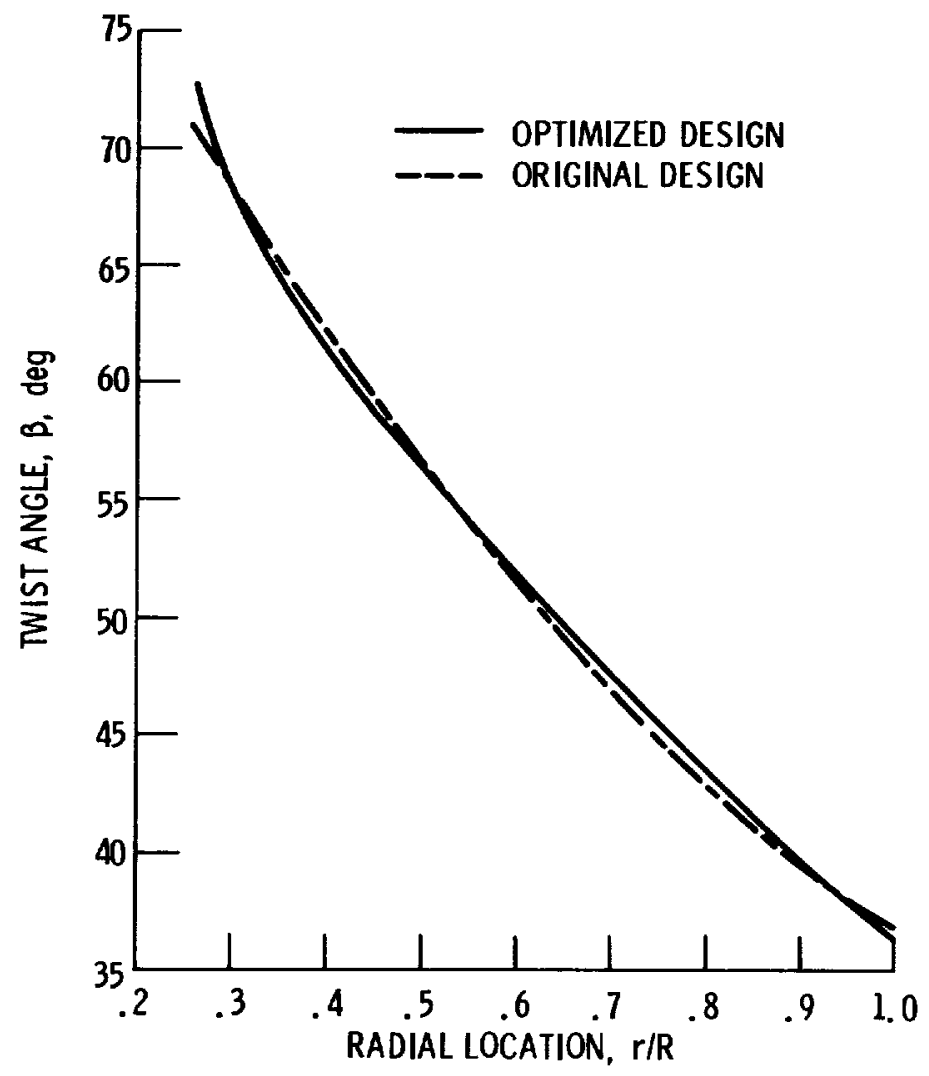

Figure 8 - Comparison of the original and optimized blade-twist for the lower speed propeller. Freestream Mach number, 0.44; two blade propeller; advance ratio, 2. 2; blade angle at 0.75 radius, $45^{\circ}$. 


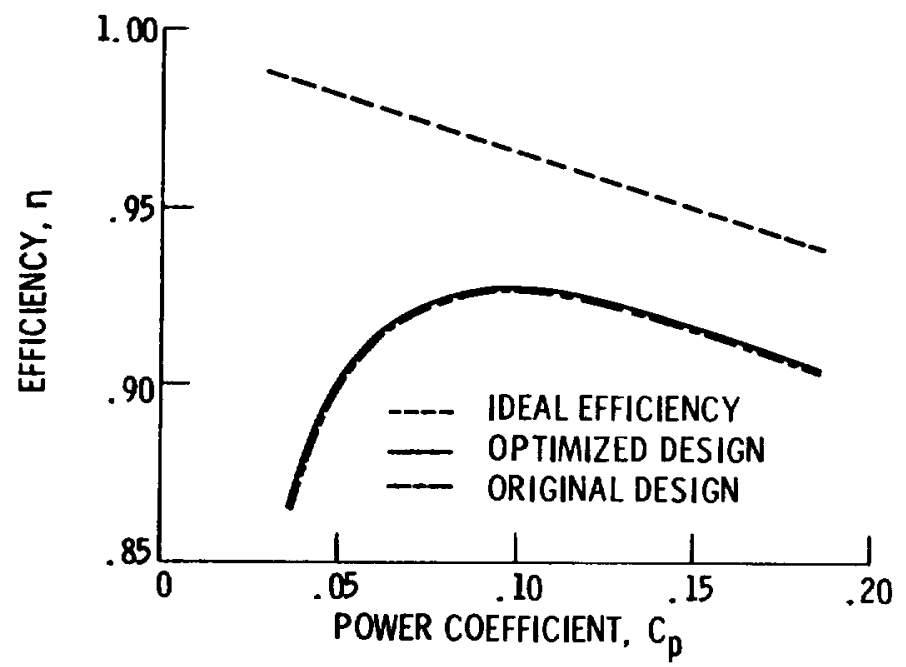

Figure 9. - Comparison of predicted efficiencies for the lower speed propeller. Freestream Mach number. 0.44; two blade propeller; advance ratio, 2.2; blade angle at 0.75 radius, $45^{\circ}$.

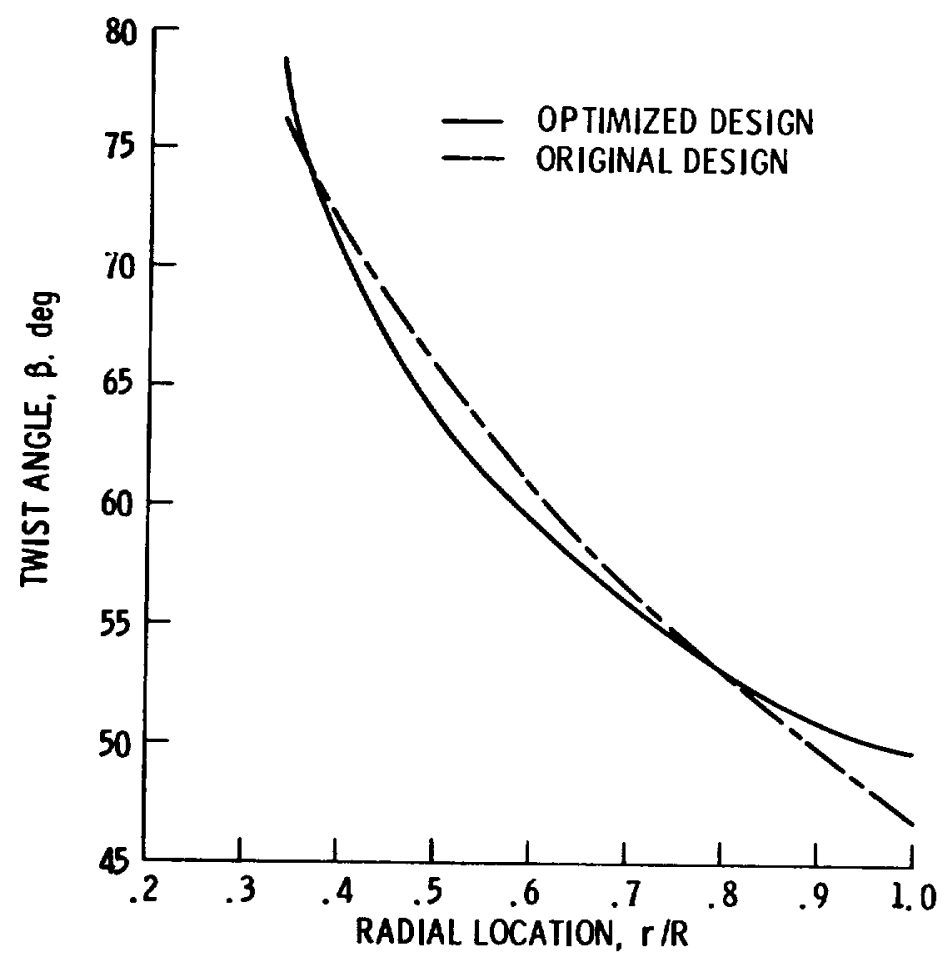

Figure 10. - Comparison of the original and optimized blade-twist for the higher speed propeller. Freestream Mach number, 0.71; two blade propeller: advance ratio, 3.3 ; blade angle at 0.75 radius, $55^{\circ}$. 


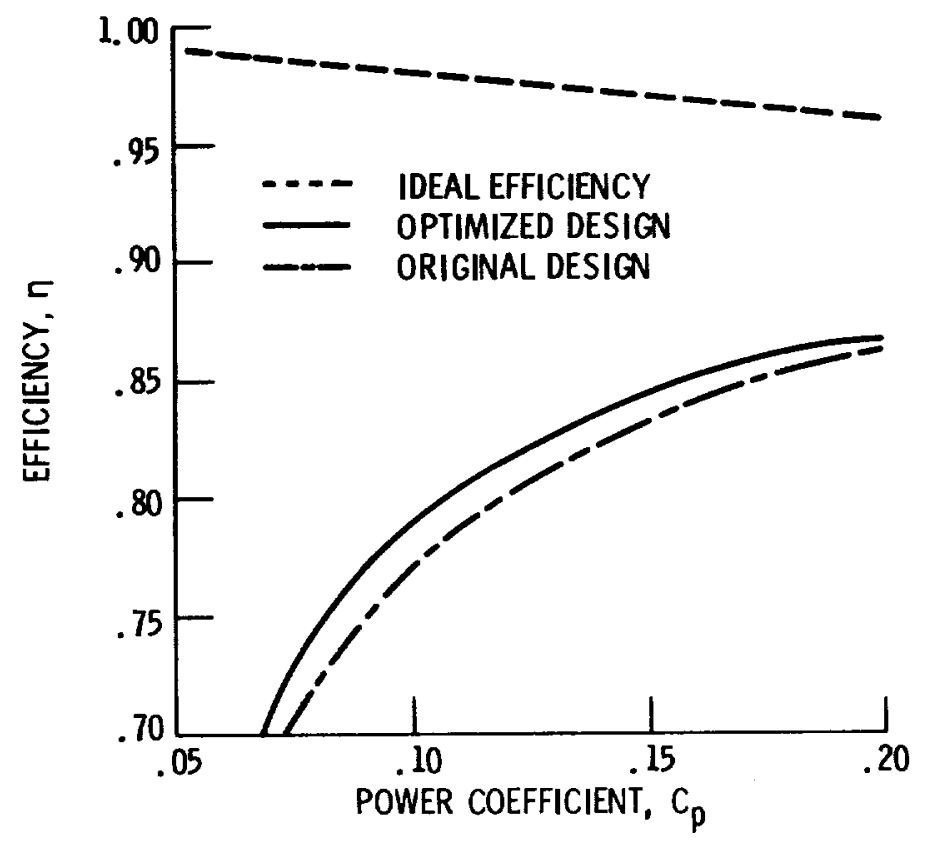

Figure 11. - Comparison of predicted efficiencies for the higher speed propeller. Freestream Mach number, 0.71 ; two blade propeller; advance ratio, 3. 3; blade angle at 0.75 radius, $55^{\circ}$. 



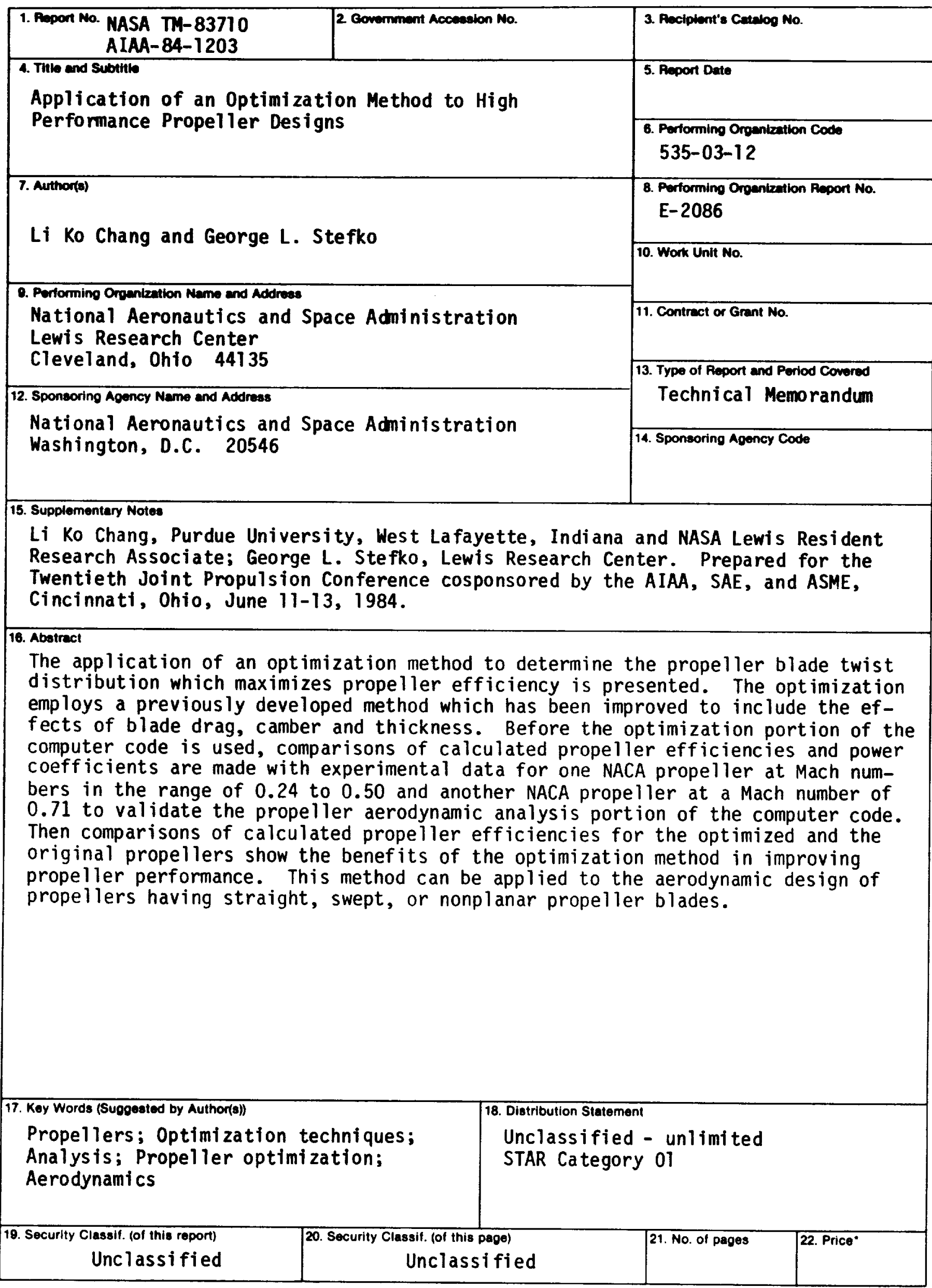

"For sale by the National Technical Intormation Service. Springfield, Virginia 22161 


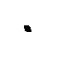


Eecial fouATH Class mall

cook
20546

Official Business

Penaliy for Private Use, $\$ 300$
National Aeronautics and
Space Administration
Washington, D.C.

National Aeronautics
Space Administration
Washington, D.C.

National Aeronauti
Space Administratic
Washington, D.C.

.

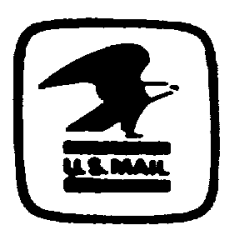

Ponece and Fees Paid National Acronouticas end Spece Administration MASA-451 